\title{
Reduced intensity conditioning before allografting: moderate enthusiasm may be more appropriate
}

\author{
U. Schuler
}

Since the first clinical series of allogeneic bone marrow transplants were reported, the procedure has been associated with a considerable amount of pulmonary complications, among many other problems. Over the last three decades, huge efforts have been devoted to the avoidance and the effective treatment of these complications. In the past, numerous patients did not undergo transplantation, either because they were considered too ill or too old to tolerate these procedure-related side-effects. In this issue of the European Respiratory Journal (ERJ), NUSAIR et al. [1] from Jerusalem report on their experience with pulmonary complications as observed after transplants with "nonmyeloablative" conditioning.

Stem cell transplantation (SCT) can be divided into three components: the conditioning therapy (chemotherapy with/ without irradiation), the source and composition of the graft, and the post-transplant immunosuppression. Modifications of all three components may contribute to the profile of efficacy and toxicity of the procedure. The group from the Hadassah University Hospital in Jerusalem was among the first to develop a procedure that tried to minimise the toxicity of the preparative regimen and relied more on the graftversus-leukaemia (GVL) effect of the transplanted allogeneic immune system [2]. Numerous other centres then followed and this has resulted in the emergence of a variety of different conditioning regimes, almost a continuum from purely immunosuppressive to conventional dosage being individualised by drug monitoring. Terminology is not uniform, the approach has also been designated "dosereduced conditioning" or "reduced intensity conditioning", as many transplant physicians felt that "nonmyeloablative conditioning" was a misnomer of a procedure that results in complete lymphohaematopoietic donor chimerism in the majority of recipients.

Given the relative success of established conditioning regimens, it is no surprise that these new strategies were not developed with the same groups of patients in mind. On the one hand, there was interest in an adoptive immunotherapy in patients whose tumour would not benefit from any of the established conditioning regimens, e.g. patients with solid tumours susceptible to immunotherapy (such as renal cell carcinoma). On the other hand, there are patients with classical indications for SCT, e.g. acute leukaemia, who are not regarded eligible for the conventional procedure, most commonly because of advanced age or toxicities or infections from preceding chemotherapies. In the latter group, the obvious fear would be that, by reducing the intensity of the anti-leukaemic chemotherapy, the benefit of the procedure would be reduced, unless the immunologic GVL effect

Correspondence: U. Schuler, Medizinische Klinik und Poliklinik I, Universitätsklinikum Carl Gustav Carus, Technische Universität Dresden, Fetscherstrasse 74, D-01307 Dresden, Germany. Fax: 49 3514585362. E-mail: Ulrich.Schuler@uniklinikum-dresden.de compensated for this loss of effect. Unfortunately, the true value of the procedure is, as yet, difficult to delineate. The notion of "noneligibility" for a conventional transplant is not clearly defined. If the notion makes sense, it obviously makes the request for a randomised comparison between reduced intensity conditioning and conventional conditioning unethical, at least if the distinction was absolutely clear. The concept of noneligibility may also change over time, e.g. a history of aspergillosis in the era of liposomal amphotericin and voriconazole need not be as prohibitive for a conventional transplant as it used to be 10 yrs earlier. Not unexpectedly, one systematic review [3] could only describe a number of heterogeneous, small prospective case series, most of which "lacked concurrent or historical controls" and in which "outcomes of interest were not uniformly reported".

It has become a frequently quoted one-liner that "trials with controls tend to lack enthusiasm, while trials with enthusiasm tend to lack controls". One has to keep in mind that enthusiasm about good treatment results in small series may be a source of publication bias, as good and surprising results are published more easily. Indeed, the early case series about nonmyeloablative SCT generated a lot of enthusiasm, e.g. when the survival of the first 26 patients was described as follows: "with an observation period extending over $1 \mathrm{yr}$ (median, 8 months), 22 of 26 patients $(85 \%)$ treated by allogeneic nonmyeloablative stem cell transplantation are alive, $21(81 \%)$ disease-free by all measurable criteria....., with excellent quality of life, and a Karnofsky score of 100\%" [2]. The present report from the same group in this issue of the $E R J$ [1], concerning 53 patients with an identical conditioning (and probably with a considerable overlap of patients with the first series), shows a death rate of 34\% (18 out of 53) at day 100 , a time when the disease-free-survival plot of the first publication suggests a death rate of 0 [2]. Although 10 of these 18 deaths were classified as transplant related, one is inclined to follow the authors in their argument, which states that the treatment-related morbidity and mortality overall is lower than expected if the risk profile of the patient population is taken into account.

However, the optimism to regard reduced-intensity conditioning SCT as a panacea has waned. Controlled or, at least, disease-specific studies are warranted (and on the way). Given the fact that GVL is the mainstay of therapy, it would be a surprise to have much less than expected graft-versushost disease (GVHD). Interestingly, the distinction between acute and chronic forms of GVHD seems to be blurred, with clinically "acute" forms of GVHD occurring much later than usual [4]. Accordingly, reports about higher incidences of complications associated with GVHD and the ensuing immunosuppression, such as fungal and cytomegaly virus infections, are emerging [5-9]. In the absence of randomised trials, disease-specific survival rates should be considered in preference to the mixed bag of treatment-specific outcomes with heterogeneous diseases used in most studies. These 
studies usually show that, although control of the underlying malignancy is possible in some cases, rapidly proliferating disease is not cured in the majority of patients [10].

At present, two areas of development seem the most promising: first, treatment of the more indolent and slowly progressing forms of malignancy, such as chronic lymphocytic leukaemia [11, 12]; and secondly, consolidative allogeneic transplantation in acute leukaemias in remission. The latter is the area that allows for randomised comparisons, as there are, presumably, a sufficient number of patients who are neither too ill nor ideal candidates for conventional conditioning.

\section{References}

1. Nusair S, Breuer R, Shapira MY, Berkman N, Or R. Low incidence of pulmonary complications following nonmyeloablative stem cell transplantation. Eur Respir J 2004; 23: $440-445$.

2. Slavin S, Nagler A, Naparstek E, et al. Nonmyeloablative stem cell transplantation and cell therapy as an alternative to conventional bone marrow transplantation with lethal cytoreduction for the treatment of malignant and nonmalignant hematologic diseases. Blood 1998; 91: 756-763.

3. Djulbegovic B, Seidenfeld J, Bonnell C, Kumar A. Nonmyeloablative allogeneic stem-cell transplantation for hematologic malignancies: a systematic review. Cancer Control 2003; 10: 17-41.

4. Mielcarek M, Martin PJ, Leisenring W, et al. Graft-versushost disease after nonmyeloablative versus conventional hematopoietic stem cell transplantation. Blood 2003; 102: 756-762.

5. Hagen EA, Stern $\mathrm{H}$, Porter D, et al. High rate of invasive fungal infections following nonmyeloablative allogeneic transplantation. Clin Infect Dis 2003; 36: 9-15.

6. Chakrabarti S, Mackinnon S, Chopra R, et al. High incidence of cytomegalovirus infection after nonmyeloablative stem cell transplantation: potential role of Campath- $1 \mathrm{H}$ in delaying immune reconstitution. Blood 2002; 99: 43574363.

7. Mohty M, Jacot W, Faucher C, et al. Infectious complications following allogeneic HLA-identical sibling transplantation with antithymocyte globulin-based reduced intensity preparative regimen. Leukemia 2003; 17: 2168-2177.

8. Schetelig J, Oswald O, Steuer N, et al. Cytomegalovirus infections in allogeneic stem cell recipients after reducedintensity or myeloablative conditioning assessed by quantitative PCR and pp65-antigenemia. Bone Marrow Transplant 2003; 32: 695-701.

9. Fukuda T, Boeckh M, Carter RA, et al. Risks and outcomes of invasive fungal infections in recipients of allogeneic hematopoietic stem cell transplants after nonmyeloablative conditioning. Blood 2003; 102: 827-833.

10. Wong R, Giralt SA, Martin T, et al. Reduced-intensity conditioning for unrelated donor hematopoietic stem cell transplantation as treatment for myeloid malignancies in patients older than 55 years. Blood 2003; 102: 3052-3059.

11. Schetelig J, Thiede C, Bornhauser M, et al. Evidence of a graft-versus-leukemia effect in chronic lymphocytic leukemia after reduced-intensity conditioning and allogeneic stem-cell transplantation: the Cooperative German Transplant Study Group. J Clin Oncol 2003; 21: 2747-2753.

12. Dreger P, Brand R, Hansz J, et al. Treatment-related mortality and graft-versus-leukemia activity after allogeneic stem cell transplantation for chronic lymphocytic leukemia using intensity-reduced conditioning. Leukemia 2003; 17: 841-848. 